MATHEMATICS OF COMPUTATION

Volume 69, Number 230 , Pages 721-747

S 0025-5718(99)01107-2

Article electronically published on February 24, 1999

\title{
ON THE CONVERGENCE \\ OF CERTAIN GAUSS-TYPE QUADRATURE FORMULAS FOR UNBOUNDED INTERVALS
}

\author{
A. BULTHEEL, C. DÍAZ-MENDOZA, P. GONZÁLEZ-VERA, AND R. ORIVE
}

Dedicated to Professor Nácere Hayek Calil on the occasion of his 75th birthday

\begin{abstract}
We consider the convergence of Gauss-type quadrature formulas for the integral $\int_{0}^{\infty} f(x) \omega(x) \mathrm{d} x$, where $\omega$ is a weight function on the half line $[0, \infty)$. The $n$-point Gauss-type quadrature formulas are constructed such that they are exact in the set of Laurent polynomials $\Lambda_{-p, q-1}=\left\{\sum_{k=-p}^{q-1} a_{k} x^{k}\right\}$, where $p=p(n)$ is a sequence of integers satisfying $0 \leq p(n) \leq 2 n$ and $q=$ $q(n)=2 n-p(n)$. It is proved that under certain Carleman-type conditions for the weight and when $p(n)$ or $q(n)$ goes to $\infty$, then convergence holds for all functions $f$ for which $f \omega$ is integrable on $[0, \infty)$. Some numerical experiments compare the convergence of these quadrature formulas with the convergence of the classical Gauss quadrature formulas for the half line.
\end{abstract}

\section{INTRODUCTION}

In this paper, we consider the very classical and yet up-to-date problem of approximating a definite integral

$$
I_{\alpha}(f)=\int_{a}^{b} f(x) \mathrm{d} \alpha(x),
$$

where $\alpha$ is a distribution function, i.e. a real valued, bounded, nondecreasing function with infinitely many points of increase on $(a, b)$. Many approaches have been proposed to estimate (1.1), especially when $[a, b]$ is a finite interval and $\mathrm{d} \alpha(x) \equiv \mathrm{d} x$ on $(a, b)$.

Most of these methods yield estimates of the form

$$
I_{n}(f)=\sum_{j=1}^{n} A_{j} f\left(x_{j}\right)
$$

Received by the editor March 3, 1998 and, in revised form, May 19, 1998.

1991 Mathematics Subject Classification. Primary 65D30; Secondary 41A21.

The work of the first author is partially supported by the Fund for Scientific Research (FWO), project "Orthogonal systems and their applications", grant \#G.0278.97, and the Belgian Programme on Interuniversity Poles of Attraction, initiated by the Belgian State, Prime Minister's Office for Science, Technology and Culture. The scientific responsibility rests with the author.

The work of the other three authors was partially supported by the scientific research project PB96-1029 of the Spanish D.G.I.C.Y.T. 
with $n$ a natural number, $\left\{x_{j}\right\}_{j=1}^{n}$ points on $(a, b)$, and $\left\{A_{j}\right\}_{j=1}^{n}$ coefficients or weights, determined such that

$$
I_{\alpha}(f)=I_{n}(f)
$$

for all $f$ belonging to a certain class of functions with special features. Assume that for the distribution $\alpha$ all the moments

$$
c_{k}=\int_{a}^{b} x^{k} \mathrm{~d} \alpha(x)
$$

exist for all nonnegative integers $k$. Under this condition, it can be assured that there exist $n$ distinct nodes $x_{1}, \ldots, x_{n}$ on $(a, b)$ and $n$ positive weights $A_{1}, \ldots, A_{n}$ so that

$$
I_{\alpha}(f)=I_{n}(f)=\sum_{j=1}^{n} A_{j} f\left(x_{j}\right), \quad \forall f \in \Pi_{2 n-1},
$$

where $\Pi_{k}, k \geq 0$, denotes the space of all polynomials of degree at most $k$. This gives rise to the well known Gauss-Christoffel quadrature formulas [14, and these are studied together with the intimately related problems of orthogonal polynomials and Padé approximation. This can be seen as follows. Let $Q_{n}$ denote the $n$th orthogonal polynomial with respect to the distribution $\alpha$, i.e. $Q_{n} \in \Pi_{n}$ and

$$
\int_{a}^{b} x^{j} Q_{n}(x) \mathrm{d} \alpha(x)=0, \quad j=0,1, \ldots, n-1,
$$

while this integral is nonzero for $j=n$. Then the nodes $x_{j}$ are the zeros of $Q_{n}$. Moreover, $Q_{n}$ is the denominator of the $[n-1 / n]$ Padé approximant at $\infty$ of the Cauchy transform $F_{\alpha}$ of the distribution $\alpha$ :

$$
F_{\alpha}(z)=\int_{a}^{b} \frac{\mathrm{d} \alpha(x)}{z-x}
$$

Note that $F_{\alpha}$ has the asymptotic expansion

$$
L_{\infty}(z)=\sum_{j=1}^{\infty} c_{j-1} z^{-j}, \quad z \rightarrow \infty,
$$

and the $[n-1 / n]$ Padé approximant is of the form $P_{n-1} / Q_{n}$ with $P_{n-1} \in \Pi_{n-1}$, and it is defined by the condition

$$
F_{\alpha}(z)-\frac{P_{n-1}(z)}{Q_{n}(z)}=O\left(z^{-(2 n+1)}\right), \quad z \rightarrow \infty .
$$

The convergence of the sequence $\left\{I_{n}(f)\right\}$ to the integral $I_{\alpha}(f)$ for all functions $f$ in a class that is as large as possible is a problem that was almost completely solved in the case of a finite interval $(a, b)$ by Stieltjes [21. However, the case of an infinite interval needs special care. A first contribution was given by Stieltjes in [22], where the special case $f(x)=1 /(z-x)$ is considered for $z \notin[a, b)=[0, \infty)$.

Other contributions in this field are the papers by Uspenski in 1916 [23] and 1928 [24. Further details, as well as a long list of references, can be found in the comprehensive survey by Gautschi [9] and the book by Davis and Rabinowitz [7].

In the sequel we shall assume for the sake of simplicity that $\alpha$ is absolutely continuous on $(a, b)$, so that we have

$$
\mathrm{d} \alpha(x)=\omega(x) \mathrm{d} x
$$


with $\omega(x)>0$ almost everywhere on $(a, b)$, and we shall write

$$
I_{\alpha}(f)=I_{\omega}(f)=\int_{a}^{b} f(x) \omega(x) \mathrm{d} x, \quad 0 \leq a<b \leq+\infty .
$$

If $\alpha$ is not absolutely continuous, then the Riemann integrals should be replaced by Riemann-Stieltjes integrals, but the results are basically the same. Furthermore, we shall assume that all the moments

$$
c_{k}=\int_{a}^{b} x^{k} \omega(x) \mathrm{d} x
$$

exist for $k=0, \pm 1, \pm 2, \ldots$ This gives rise to quadrature formulas like (1.2) integrating exactly, not only polynomials, but more general functions, namely the Laurent polynomials (or L-polynomials), which are given by

$$
\Lambda_{p, q}=\left\{L(z)=\sum_{j=p}^{q} \beta_{j} z^{j}\right\}, \quad p, q \in \mathbb{Z}, \quad p \leq q .
$$

As far as we know, quadrature formulas valid in certain subspaces of $\Lambda$, the space of all Laurent polynomials, were first introduced by Jones, Thron and Waadeland in connection with the strong Stieltjes moment problem [13].

In a similar way as for the Gauss formulas, two topics immediately arise here: the orthogonal Laurent polynomials (or equivalently orthogonal polynomials with respect to a varying weight function on intervals contained in $[0, \infty)$ ) and two-point approximants to

$$
F_{\omega}(z)=\int_{a}^{b} \frac{\omega(x)}{z-x} \mathrm{~d} x
$$

in the points 0 and $\infty$. Now, $F_{\omega}$ allows two asymptotic expansions: one at the origin and one at $\infty$ :

$$
L_{0}=\sum_{j=0}^{\infty} c_{-(j+1)} z^{j}(z \rightarrow 0) ; \quad L_{\infty}=\sum_{j=1}^{\infty} c_{j-1} z^{-j},(z \rightarrow \infty) .
$$

Among the most relevant works in this field, we can mention the contributions by W.B. Jones, O. Njåstad and W. Thron [12, 11, L. Cochran and S. Cooper [6], S. Ranga [19], and G. López-Lagomasino [15].

Very deep investigations of the special case $f(z)=1 /(z-x)$ with $z \notin(0, \infty)$, a parameter, were given in [15] as a compilation of several papers [16, 17, 18] by LópezLagomasino. His results were derived in the framework of Padé approximation for meromorphic functions of Stieltjes type. This work can be considered as the unbounded equivalent of Stieltjes' work [21].

The present paper can also be considered as a continuation of the previous papers [4, 2, 3] by the present authors. In 3] we proved, under appropriate conditions, the convergence of the quadrature formulas $I_{n}(f)$ to $I_{\omega}(f)$ for any function $f$ in the class $C^{B}[0, \infty)$ of continuous functions on $[0, \infty)$ such that $\lim _{x \rightarrow+\infty} f(x)$ exists and is finite. The aim of this paper is to extend the convergence to a class of functions $f$ which is larger than $C^{B}[0, \infty)$. We shall make use of the ideas used by Uspensky in [24].

The paper has the following structure: first we give some preliminary results in Section [2] then Section 3 gives the results about convergence, and in Section 4 we give some numerical examples. 


\section{Preliminary Results}

Throughout the remainder of the paper, we shall deal with the unbounded interval $(a, b)=(0, \infty)$, so that we shall treat the integral

$$
I_{\omega}(f)=\int_{0}^{\infty} f(x) \omega(x) \mathrm{d} x
$$

where $\omega$ is a weight function on $(0, \infty)$ such that the moments

$$
c_{k}=\int_{0}^{\infty} x^{k} \omega(x) \mathrm{d} x
$$

exist for all integer $k$.

Let $p$ and $n$ be nonegative integers with $0 \leq p \leq 2 n$. Then it can be proved (see e.g. 2] ) that there exist nodes $x_{1, n}, x_{2, n}, \ldots, x_{n, n}\left(x_{j, n} \neq x_{i, n}\right.$ for $\left.i \neq j\right)$ in $(0, \infty)$ and positive weights $A_{1, n}, A_{2, n}, \ldots, A_{n, n}$ such that

$$
I_{n}(f):=\sum_{j=1}^{n} A_{j, n} f\left(x_{j, n}\right)=I_{\omega}(f), \quad \forall f \in \Lambda_{-p, 2 n-1-p} .
$$

We refer to $I_{n}(f)$ as the $n$-point Gauss-type quadrature formula for the subspace $\Lambda_{-p, 2 n-1-p \text {. }}$

Set $Q_{n}(x)=\gamma_{n}\left(x-x_{1, n}\right) \cdots\left(x-x_{n, n}\right)\left(\gamma_{n} \neq 0\right)$; then it is known that $Q_{n}(x)$ represents the $n$th orthogonal polynomial with respect to $x^{-p} \omega(x)$, i.e.,

$$
\int_{0}^{\infty} x^{j} Q_{n}(x) \frac{\omega(x)}{x^{p}} \mathrm{~d} x=0, \quad j=0,1, \ldots, n-1 .
$$

Furthermore, $Q_{n}$ is the denominator of the $[p / n]$ two-point Padé approximant (2PA) in the points 0 and $\infty$ for the Cauchy transform

$$
F_{\omega}(z)=\int_{0}^{\infty} \frac{\omega(x)}{z-x} \mathrm{~d} x .
$$

This means that there exists a unique polynomial $P_{n-1} \in \Pi_{n-1}$ such that the rational function $P_{n-1}(z) / Q_{n}(z)$ satisfies

$$
\begin{aligned}
& F_{\omega}(z)-\frac{P_{n-1}(z)}{Q_{n}(z)}=O\left(z^{p}\right), \quad z \rightarrow 0, \\
& F_{\omega}(z)-\frac{P_{n-1}(z)}{Q_{n}(z)}=O\left(z^{-(2 n-p+1)}\right), \quad z \rightarrow \infty .
\end{aligned}
$$

For further details concerning 2PA, we refer to [4. Observe that when we take $p=0,2 \mathrm{PA}$ at $z=0$ and $z=\infty$ become one point Padé approximants at $z=\infty$ and thus the classical Gauss-Christoffel quadrature formulas arise as a special case.

For a given sequence $\{p(n)\}_{1}^{\infty}$ of nonnegative integers such that $0 \leq p(n) \leq 2 n$, we are interested in convergence of the Gauss-type formula $I_{n}(f)$ in $\Lambda_{-p(n), 2 n-1-p(n)}$ to $I_{\omega}(f)$ as $n \rightarrow \infty$. This convergence should hold for all $f$ in a class of functions that is as large as possible.

Some preliminary results for $p(n)=n$ were given by Jones et al. 11] in the case of a bounded interval. As for the classical case $(p(n)=0$ for all $n)$, an infinite interval is more difficult than a finite one. In the work of López-Lagomasino [15], 
some Carleman type conditions on the moments $c_{k}$ and conditions on the numbers $p(n)$ were assumed, namely

$$
\lim _{n \rightarrow \infty} 2 n-p(n)=\infty \quad \text { and } \quad \sum_{j=1}^{\infty} c_{j}^{-\frac{1}{2 j}}=\infty
$$

or

$$
\lim _{n \rightarrow \infty} p(n)=\infty \quad \text { and } \quad \sum_{j=1}^{\infty} c_{-j}^{-\frac{1}{2 j}}=\infty .
$$

Under these conditions, it can be deduced (see [3]) that the corresponding sequence of Gauss-type formulas $I_{n}(f)$ in $\Lambda_{-p(n), 2 n-1-p(n)}$ will converge to $I_{\omega}(f)$ for any function $f$ in the class $C^{B}[0, \infty)$ of continuous functions on $[0, \infty)$ such that $\lim _{x \rightarrow+\infty} f(x)$ exists and is finite. To extend the convergence to a larger class, we need some preliminary results.

Theorem 2.1 (see [3). Let $\{p(n)\}$ be a sequence of integers such that $0 \leq p(n) \leq$ $2 n$. Let $\omega$ be a weight function on $[0, \infty)$ and let $I_{n}(f)=\sum_{j=1}^{n} A_{j, n} f\left(x_{j, n}\right)$ denote the corresponding n-point Gauss-type formula for $\Lambda_{-p(n), 2 n-1-p(n)}$. Define $Q_{n}(x)=$ $\left(x-x_{1, n}\right) \cdots\left(x-x_{n, n}\right)$. Then, if $f \in C^{(2 n)}[0, \infty)$,

$$
I_{\omega}(f)=I_{n}(f)+\frac{\gamma_{n}}{(2 n) !}\left[x^{p(n)} f(x)\right]_{x=\theta_{n}}^{(2 n)},
$$

where $\theta_{n} \in[0, \infty)$ and

$$
\gamma_{n}=\int_{0}^{\infty} Q_{n}(x)^{2} \frac{\omega(x)}{x^{p(n)}} \mathrm{d} x .
$$

Remark 2.1. Clearly, this theorem implies that

$$
I_{n}(L)=I_{\omega}(L), \quad \forall L \in \Lambda_{-p(n), q(n)-1},
$$

where $p(n)+q(n)=2 n$.

Observe that the classical polynomial case corresponds to $p(n)=0$ for all $n$. The case $p(n)=2 n$ is similar because a simple change of variables $x \rightarrow 1 / x$ will reduce it to the polynomial case. To deal with quadrature formulas which are valid in subspaces of proper Laurent polynomials, we will assume in the sequel that $0 \leq p(n)<2 n$

Furthermore, we recall that the weights $A_{j, n}$ are positive, and, because $1 \epsilon$ $\Lambda_{-p(n), 2 n-1-p(n)}$ for any $n$, one has

$$
\sum_{j=1}^{n} A_{j, n}=c_{0}, \quad n=1,2, \ldots
$$

We will now give extensions of the Chebyshev inequalities [5] that are applicable in our situation. Therefore, we need two lemmas.

Lemma 2.2. Let $\tilde{p}, k$ and $n$ be integers such that $0 \leq \tilde{p} \leq 2 n-1$ and $1 \leq k \leq n-1$, and set $\tilde{q}=2 n-2-\tilde{p}$. Consider the positive numbers $\left\{x_{j}\right\}_{j=1}^{n}$, ordered so that $0<x_{1}<x_{2}<\cdots<x_{n}$. Then there exists an $L \in \Lambda_{-\tilde{p}, \tilde{q} \text { satisfying }}$

1. $L\left(x_{i}\right)=1, \quad i=1, \ldots, k$,

2. $L\left(x_{i}\right)=0, \quad i=k+1, \ldots, n$,

3. $L(x) \geq 0, \quad \forall x>0$, 


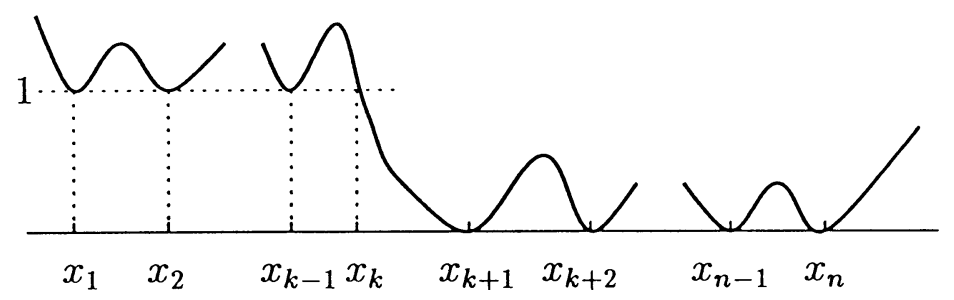

Figure 1. The function $L(x)$

4. $L(x) \geq 1, \quad \forall x \in\left(0, x_{k}\right]$

Proof. Take into account that $\operatorname{dim}\left(\Lambda_{-\tilde{p}, \tilde{q}}\right)=\tilde{p}+\tilde{q}+1=2 n-1$ since $\tilde{q}=2 n-2-\tilde{p}$. Thus, there exists a unique $L \in \Lambda_{-\tilde{p}, \tilde{q}}$ satisfying the interpolation conditions (all the $x_{i}$ are nonzero and different from each other)

$$
\begin{aligned}
L\left(x_{i}\right) & =1, \quad i=1, \ldots, k, \\
L\left(x_{i}\right) & =0, \quad i=k+1, \ldots, n, \\
L^{\prime}\left(x_{i}\right) & =0, \quad i=1, \ldots, n, i \neq k .
\end{aligned}
$$

We now prove that this $L$ satisfies the requirements of the lemma.

For $\tilde{p}=0$, we have the polynomial situation, and in this case the lemma is known. It can be found for example in [5].

Thus assume $0<\tilde{p}$. Using Rolle's theorem, it can be seen that $L^{\prime}(x)$ vanishes at at least $2 n-3$ points on $\left(0, x_{n}\right)$.

If $\tilde{p}=2 n-2$, then $L^{\prime}(x)=x^{-(\tilde{p}+1)} \tilde{P}(x), \tilde{P} \in \Pi_{2 n-3}$; thus $L^{\prime}(x)$ has exactly $2 n-3$ zeros in $\left(0, x_{n}\right)$, and it can only behave as in Figure 1. Therefore $L(x)$ satisfies the conditions of the lemma.

If $\tilde{p} \neq 2 n-2$, then $L^{\prime}(x)=x^{-(\tilde{p}+1)} \tilde{Q}(x)$, with $\tilde{Q}(x) \in \Pi_{2 n-2}$. Thus $L^{\prime}$ has $2 n-2$ zeros, of which $2 n-3$ are as in Figure 1. Our aim is to prove that the remaining zero of $L^{\prime}(x)$ cannot be on $\left(0, x_{n}\right)$. This is immediate for $\tilde{p}>2 n-2$ because $L(\infty)=0$ and $L\left(x_{n}\right)=0$, so that there should exist some $\xi>x_{n}$ such that $L^{\prime}(\xi)=0$. Finally, assume $0<\tilde{p}<2 n-2$. We know that $\tilde{Q}(x)$ has at least $2 n-3$ positive zeros. If the other one were positive too, then we know that by the Cardan-Vieta formulas, the coefficients of $\tilde{Q}(x)$ should all be nonzero. Set for $0<\tilde{p} \leq 2 n-3$

$$
L(x)=x^{-\tilde{p}} P(x), \quad P(x)=\sum_{j=0}^{2 n-2} b_{j} x^{j} \in \Pi_{2 n-2} .
$$

Hence

$$
L^{\prime}(x)=x^{-(\tilde{p}+1)} \tilde{Q}(x), \quad \tilde{Q}(x)=-\tilde{p} P(x)-x P^{\prime}(x)=\sum_{j=0}^{2 n-2} a_{j} x^{j},
$$

where $a_{0}=-\tilde{p} b_{0}$ and $a_{j}=b_{j}(j-\tilde{p})$ for $1 \leq j \leq 2 n-2$. Thus $a_{\tilde{p}}=0$, which is a contradiction. Therefore, the remaining zero in the case $1 \leq \tilde{p} \leq 2 n-3$ should be negative. We thus find that the behavior of $L$ is again as in Figure 1

The following lemma can be proved in a similar way. 
Lemma 2.3. Let $\tilde{p}, k$ and $n$ be integers satisfying $0 \leq \tilde{p} \leq 2 n-1$ and $1 \leq k \leq n-1$, and define $\tilde{q}$ by $\tilde{p}+\tilde{q}=2 n-2$. Let $0<x_{1}<x_{2}<\cdots<x_{n}$ be real numbers. Then there exists an $R \in \Lambda_{-\tilde{p}, \tilde{q}}$ satisfying the conditions

1. $R\left(x_{i}\right)=1, \quad i=1, \ldots, k$,

2. $R\left(x_{i}\right)=0, \quad i=k+1, \ldots, n$,

3. $R(x) \leq 0, \quad \forall x \geq x_{k+1}$,

4. $R(x) \leq 1, \quad \forall x>0$.

Now we can state the following

Theorem 2.4 (Chebyshev inequality). Let $I_{n}(f)=\sum_{j=1}^{n} A_{j, n} f\left(x_{j, n}\right)$ be the $n$ point Gauss-type formula in $\Lambda_{-\tilde{p}, 2 n-1-\tilde{p}}(0 \leq \tilde{p} \leq 2 n-1)$ with respect to the weight function $\omega(x)$. Then

$$
\int_{0}^{x_{k, n}} \omega(x) \mathrm{d} x<A_{1, n}+A_{2, n}+\cdots+A_{k, n}<\int_{0}^{x_{k+1, n}} \omega(x) \mathrm{d} x,
$$

where $k$ is a fixed integer such that $1 \leq k \leq n-1$.

Proof. Take the Laurent polynomial $L$ as in Lemma 2.2. where the points $x_{j}$ are replaced by the nodes of the quadrature formula. Because $L \in \Lambda_{-\tilde{p}, 2 n-1-\tilde{p} \text {, the }}$ quadrature is exact for $L$, so that

$$
\int_{0}^{\infty} L(x) \omega(x) \mathrm{d} x=\sum_{j=1}^{n} A_{j, n} L\left(x_{j, n}\right)=\sum_{j=1}^{k} A_{j, n} .
$$

Thus

$$
\int_{0}^{x_{k, n}} \omega(x) \mathrm{d} x \leq \int_{0}^{x_{k, n}} L(x) \omega(x) \mathrm{d} x<\sum_{j=1}^{k} A_{j, n} .
$$

On the other hand, using the L-polynomial $R$ of Lemma 2.3 we obtain in a similar way that

$$
\sum_{j=1}^{k} A_{j, n}<\int_{0}^{x_{k+1, n}} R(x) \omega(x) \mathrm{d} x \leq \int_{0}^{x_{k+1, n}} \omega(x) \mathrm{d} x .
$$

This proves the theorem.

\section{Convergence}

We start this section with a general result on convergence of Gauss-type quadrature formulas which is inspired by the work of Stieltjes [22].

Theorem 3.1. Let $\{p(n)\}$ be a sequence of nonnegative integers with $0 \leq p(n) \leq$ $2 n-1$. Let $I_{n}(f)=\sum_{j=1}^{n} A_{j, n} f\left(x_{j, n}\right), n=1,2, \ldots$, be the sequence of Gauss-type formulas in $\Lambda_{-p(n), q(n)-1}$, where $p(n)+q(n)=2 n$. Then $\lim _{n \rightarrow \infty} I_{n}(f)=I_{\omega}(f)$ for any $f$ such that $f(x) \omega(x)$ is integrable on $[0, \infty)$, if and only if $\lim _{n \rightarrow \infty} A_{j, n}=0$ uniformly in $j$.

Proof. " $\Leftarrow$ " Assume that $\lim _{n \rightarrow \infty} A_{j, n}=0$ uniformly in $j$, and take any $f$ such that $f(x) \omega(x)$ is integrable on $[0, \infty)$. Set $h(x)=\int_{0}^{x} \omega(t) \mathrm{d} t$. Since $\omega(x)>0$ a.e., this $h(x)$ is strictly increasing in $(0, \infty)$ and $h(\infty)=\int_{0}^{\infty} \omega(t) \mathrm{d} t=c_{0}$. Define $y_{j, n}=h\left(x_{j, n}\right), j=1, \ldots, n ; n=1,2, \ldots$ Then, by Theorem 2.4 it is seen that

$$
0<y_{1, n}<A_{1, n}<y_{2, n}<A_{1, n}+A_{2, n}<y_{3, n}<A_{1, n}+A_{2, n}+A_{3, n}<\cdots .
$$


Since $h(x)$ is continuous, there exist $\theta_{j, n}$ such that

$$
A_{1, n}+A_{2, n}+\cdots+A_{j, n}=h\left(\theta_{j, n}\right)=: \tilde{y}_{j, n}
$$

and $\tilde{y}_{j+1, n}-\tilde{y}_{j, n}=A_{j, n} \rightarrow 0$ uniformly in $j$. Now the function $\tilde{f}(y)=f\left(h^{-1}(y)\right)$ is integrable on $\left[0, c_{0}\right]$, and since $\sum_{j=1}^{n} \tilde{f}\left(y_{j, n}\right)\left(\tilde{y}_{j+1, n}-\tilde{y}_{j, n}\right)$ is a Riemann sum on $\left[0, c_{0}\right]$, we can write

$$
\begin{aligned}
I_{\omega}(f) & =\int_{0}^{\infty} f(y) \omega(x) \mathrm{d} x=\int_{0}^{c_{0}} \tilde{f}(y) \mathrm{d} y \\
& =\lim _{n \rightarrow \infty} \sum_{j=1}^{n} \tilde{f}\left(y_{j, n}\right)\left(\tilde{y}_{j+1, n}-\tilde{y}_{j, n}\right)=\lim _{n \rightarrow \infty} \sum_{j=1}^{n} A_{j, n} f\left(x_{j, n}\right)=\lim _{n \rightarrow \infty} I_{n}(f) .
\end{aligned}
$$

" $\Rightarrow$ " Let us take $f(x)=\chi_{[a, b]}(x)$, the characteristic function for an arbitrary interval $[a, b] \subset[0, \infty)$, i.e.,

$$
f(x)=\chi_{[a, b]}(x)= \begin{cases}1, & x \in[a, b], \\ 0, & x \notin[a, b] .\end{cases}
$$

Then

$$
\lim _{n \rightarrow \infty} \sum_{a<x_{j, n}<b} A_{j, n}=\int_{a}^{b} \omega(x) \mathrm{d} x>0 .
$$

Let us assume that there exists a sequence $\{l(n)\}$ of natural numbers such that

$$
\lim _{n \rightarrow \infty} A_{l(n), n}=A \neq 0 .
$$

Recall that for any $j, 1 \leq j \leq n, A_{j, n}<\sum_{k=1}^{n} A_{k, n}=c_{0}$. Now let the sequence $x_{l(n), n}$ (or possibly a subsequence) converge to $x$, i.e. $\lim _{n \rightarrow \infty} x_{l(n), n}=x$. This $x$ is either finite or infinite. If $x$ is finite, then we can choose $\epsilon, \epsilon^{\prime}>0$ such that

$$
\int_{x-\epsilon^{\prime}}^{x+\epsilon} \omega(\xi) \mathrm{d} \xi<\frac{A}{2}
$$

and this yields a contradiction since

$$
A \leq \lim _{n \rightarrow \infty} \sum_{x-\epsilon^{\prime} \leq x_{j, n} \leq x+\epsilon} A_{j, n}=\int_{x-\epsilon^{\prime}}^{x+\epsilon} \omega(\xi) \mathrm{d} \xi .
$$

Similarly, if $x=\infty$, we choose $M>0$ such that $\int_{M}^{\infty} \omega(x) \mathrm{d} x<A / 2$, which leads again to a contradiction. Thus $\lim _{n \rightarrow \infty} A_{j, n}=0$ uniformly in $j$.

As an immediate consequence we have

Corollary 3.2. Let $p(n)$ and $I_{n}(f)$ be as in Theorem 3.1 and assume furthermore that $\lim _{n \rightarrow \infty} A_{j, n}=0$ uniformly in $j$. Let $f$ be a function satisfying either

1. $f(x)$ is integrable on any subset $[0, a] \subset[0, \infty)$ and there exist $M$ and $m$ such that

$$
|f(x)| \leq x^{m}, \quad \forall x \geq M, M \geq 0, \quad m \in \mathbb{N}
$$

or

2. $f(x)$ is integrable on any subset $[b, \infty) \subset(0, \infty)$ and there exist $k$ and $h$ such that

$$
|f(x)| \leq x^{-k}, \quad \forall x \leq h, h \geq 0, \quad k \in \mathbb{N} .
$$

Then $\lim _{n \rightarrow \infty} I_{n}(f)=I_{\omega}(f)$. 
Observe that this convergence result holds under very general conditions on the moments $c_{k}$. That is why we allow $f$ to behave like powers of $x$ in the neighborhood of 0 and $\infty$. To allow other types of behavior in these points, we need to impose more restrictions on the moments.

So our key problem boils down to the question: Under what conditions on the moments $c_{k}$ (i.e. on the weight $\omega(x)$ ) and on the sequence of integers $p(n)$ with $0 \leq p(n) \leq 2 n-1$, does it hold that $\lim _{j \rightarrow \infty} A_{j, n}=0$ uniformly in $j$ ? Here the $A_{j, n}$ are the weights for the Gauss-type quadrature formula for $\Lambda_{-p(n), q(n)-1}$ (where $p(n)+q(n)=2 n)$.

From now on, we will assume that either

$$
c_{n} \leq C \Gamma((n+\theta+1) \gamma) R^{n \gamma}, \quad n \in \mathbb{N},
$$

or

$$
c_{-n} \leq \hat{C} \Gamma((n-\hat{\theta}-1) \gamma) \hat{R}^{n \gamma}, \quad n \in \mathbb{N},
$$

where $\theta>-1, \hat{\theta}<-1, C, \hat{C}, R$ and $\hat{R}$ are positive constants, $0<\gamma \leq 2$, and $\Gamma(s)$ is the gamma function. Note that by these conditions, the moments $c_{k}$, and hence the integrals $I_{\omega}(f)$ and the quadrature formulas $I_{n}(f)$, will depend on $\gamma$.

The following theorem involves the Mittag-Leffler function, which is defined as

$$
E_{\gamma}(y)=\sum_{k=0}^{\infty} \frac{y^{k}}{\Gamma(\gamma k+1)}, \quad \forall y \in \mathbb{C} .
$$

(Note that $E_{\gamma}$ is an entire function.) Its proof follows closely the techniques used in 24 .

Theorem 3.3. Let $\omega(x)$ be a weight function whose moments satisfy condition (3.1). Let $\{p(n)\}$ and $\{q(n)\}$ be sequences of nonnegative integers such that $p(n)+$ $q(n)=2 n$ and $\lim _{n \rightarrow \infty} q(n)=\infty$. Then

$$
\lim _{n \rightarrow \infty} I_{n}\left(E_{\gamma}\left(s^{\gamma} x\right)\right)=I_{\omega}\left(E_{\gamma}\left(s^{\gamma} x\right)\right)
$$

uniformly in compact subsets of the region $\{s \in \mathbb{C}:|s|<1 / R\}$.

Proof. Set

$$
\begin{aligned}
\phi_{n}(s) & =\sum_{k=1}^{n} A_{k, n} E_{\gamma}\left(s^{\gamma} x_{k, n}\right)=\sum_{k=1}^{n} A_{k, n}\left(\sum_{m=0}^{\infty} \frac{s^{\gamma m} x_{k, n}^{m}}{\Gamma(\gamma m+1)}\right) \\
& =\sum_{m=0}^{\infty} \frac{s^{\gamma m}}{\Gamma(\gamma m+1)} \sum_{k=1}^{n} A_{k, n} x_{k, n}^{m}=\sum_{m=0}^{\infty} \frac{s^{\gamma m}}{\Gamma(\gamma m+1)} h_{m, n},
\end{aligned}
$$

where

$$
h_{m, n}=\sum_{k=1}^{n} A_{k, n} x_{k, n}^{m} \leq c_{m}, \quad m=0,1, \ldots,
$$

as follows from Theorem 2.1. Thus

$$
\left|\phi_{n}(s)\right| \leq \sum_{m=0}^{\infty} \frac{|s|^{\gamma m}}{\Gamma(\gamma m+1)} c_{m},
$$

and from (3.1) it follows that

$$
\left|\phi_{n}(s)\right| \leq C \sum_{m=0}^{\infty} \frac{\Gamma(\gamma(m+\theta+1))}{\Gamma(\gamma m+1)}(|s| R)^{m \gamma} .
$$


Now, by the Stirling formula (see e.g. [1, (6.1.37)] or [8])

$$
\Gamma(z)=e^{-z} z^{z-1 / 2}(2 \pi)^{1 / 2}\left[1+\frac{B_{1}}{z}+\frac{B_{2}}{z^{2}}+\cdots\right], \quad z \rightarrow \infty \text { in }|\operatorname{Arg}(z)|<\pi,
$$

and therefore

$$
\frac{\Gamma(\gamma(m+\theta+1))}{\Gamma(\gamma m+1)} \text { and } \frac{1}{m^{1-\gamma(\theta+1)}}
$$

are equivalent as $m \rightarrow \infty$ in the sense that their ratio tends to a nonzero finite number. Therefore the series

$$
\sum_{m=0}^{\infty} \frac{\Gamma(\gamma(m+\theta+1))}{\Gamma(\gamma m+1)}(s R)^{\gamma m}
$$

is absolutely and uniformly convergent in the region $|s|<1 / R$. Thus $\left|\phi_{n}(s)\right|$ is uniformly bounded in $s$ and $n$, so that

$$
\phi(s)=\lim _{n \rightarrow \infty} \phi_{n}(s)=\lim _{n \rightarrow \infty} \sum_{k=0}^{n} A_{k, n} E_{\gamma}\left(s^{\gamma} x_{k, n}\right)=\sum_{k=0}^{\infty} \frac{s^{\gamma k} c_{k}}{\Gamma(\gamma k+1)} .
$$

Recall that $\lim _{n \rightarrow \infty} h_{k, n}=c_{k}$ when $h_{k, n}$ is as defined above.

On the other hand (recall that $E_{\gamma}$ is an entire function, so that the series expansion converges uniformly on $[a, b]$ so that summation and integration can be interchanged in the second line below),

$$
\begin{aligned}
\int_{0}^{\infty} E_{\gamma}\left(s^{\gamma} x\right) \omega(x) \mathrm{d} x & =\lim _{b \rightarrow \infty} \int_{0}^{b}\left(\sum_{k=0}^{\infty} \frac{s^{\gamma k} x^{k}}{\Gamma(\gamma k+1)}\right) \omega(x) \mathrm{d} x \\
& =\lim _{b \rightarrow \infty}\left(\lim _{n \rightarrow \infty} \sum_{k=0}^{n} \int_{0}^{b} \frac{s^{\gamma k} x^{k}}{\Gamma(\gamma k+1)} \omega(x) \mathrm{d} x\right) \\
& =\lim _{b \rightarrow \infty} \lim _{n \rightarrow \infty} \sum_{k=0}^{n} \frac{s^{\gamma k}}{\Gamma(\gamma k+1)} \int_{0}^{b} x^{k} \omega(x) \mathrm{d} x
\end{aligned}
$$

We note that

$$
\sum_{k=0}^{\infty}\left|\frac{s^{\gamma k}}{\Gamma(\gamma k+1)} \int_{0}^{b} x^{k} \omega(x) \mathrm{d} x\right| \leq \sum_{k=0}^{\infty} \frac{|s|^{\gamma k} c_{k}}{\Gamma(\gamma k+1)}
$$

Since the series $\sum_{k=0}^{\infty} s^{\gamma k} c_{k} / \Gamma(\gamma k+1)$ converges absolutely and uniformly in $|s|<$ $1 / R$, limits can be interchanged in (3.5), giving

$$
\begin{aligned}
I_{\omega}\left(E_{\gamma}\left(s^{\gamma} x\right)\right) & =\lim _{n \rightarrow \infty} \lim _{b \rightarrow \infty} \sum_{k=0}^{n} \frac{s^{\gamma k}}{\Gamma(\gamma k+1)} \int_{0}^{b} x^{k} \omega(x) \mathrm{d} x \\
& =\sum_{k=0}^{\infty} \frac{s^{\gamma k} c_{k}}{\Gamma(\gamma k+1)} \\
& =\phi(s)=\lim _{n \rightarrow \infty} \phi_{n}(s)=\lim _{n \rightarrow \infty} I_{n}\left(E_{\gamma}\left(s^{\gamma} x\right)\right) .
\end{aligned}
$$


Remark 3.1. Note that by (3.3) $E_{2}(y)=\cosh \left(y^{1 / 2}\right)$. Thus, Theorem 3.3 with $\gamma=2$ implies that with $I_{n}(f)$ the Gauss-type formula for $\Lambda_{-p(n), q(n)-1}$ and $p(n)+q(n)=$ $2 n$, we have

$$
\lim _{n \rightarrow \infty} I_{n}\left(\cosh s x^{1 / 2}\right)=I_{\omega}\left(\cosh s x^{1 / 2}\right), \quad s \in \mathbb{C}, \quad|s|<1 / R,
$$

where convergence is uniform in compact subsets of the indicated region. On the other hand, suppose that in (3.1) we take $\gamma<2$. Then, taking into account that now

$$
\frac{\Gamma(\gamma(m+\theta+1))}{\Gamma(2 m+1)} \text { and }\left(\frac{\gamma^{\gamma}}{4}\right)^{m} \frac{1}{m^{(2-\gamma) m} m^{1-\gamma(\theta+1)}}
$$

are of the same order at infinity, i.e., their ratio tends to a nonzero finite number as $m \rightarrow \infty$, it can be shown, by paralleling the proof of Theorem 3.3, that convergence in (3.6) holds uniformly on compact subsets of $\mathbb{C}$.

We finally observe that Theorem 3.3 was proved by Uspensky [24] in the case $\gamma=2$ and $p(n)=0, n=1,2, \ldots$ (polynomial situation).

By using (3.6) and proceeding as in [24], we arrive at

Theorem 3.4. With the same notation and under the same conditions as in Theorem 3.3,

$$
\lim _{n \rightarrow \infty} A_{j, n}=0
$$

uniformly in $j$.

Proof. Define $\Phi_{n}(s)=I_{n}\left(\cosh s x^{1 / 2}\right)$ and $\Phi(s)=I_{\omega}\left(\cosh s x^{1 / 2}\right)$. By Remark 3.1 we know that $\lim _{n \rightarrow \infty} \Phi_{n}(s)=\Phi(s)$ uniformly in compact subsets of $|s|<1 / R$ and independently of $\gamma \in(0,2]$.

Next we show uniform convergence for $s$ in the region $(0, b) \times \mathbb{R}$ with $0<b<1 / R$. Therefore we should check that the sequence $\left\{\Phi_{n}(s)\right\}$ is uniformly bounded in this region, and indeed it is because $|\cosh (a+i b)| \leq \cosh a$, so that for all $s \in(0, b) \times \mathbb{R}$ we have $\left|\Phi_{n}(s)\right| \leq \Phi_{n}(\operatorname{Re} s) \leq \Phi_{n}\left(b^{\prime}\right)$, where $b^{\prime}<b<1 / R$.

Now, for any integer $p>0$ and real $a, 0<a<1 / R$,

$$
\frac{1}{2 \pi i} \int_{a-i \infty}^{a+i \infty} \frac{e^{w s}}{s^{1+p}} \mathrm{~d} s= \begin{cases}w^{p} / \Gamma(1+p), & w>0, \\ 0, & w \leq 0,\end{cases}
$$

so that for $t>0$ (interchanging integration and summation is justified by Fubini's theorem and the fact that $|\Phi(s)| \leq \Phi(a))$

$$
\frac{1}{2 \pi i} \int_{a-i \infty}^{a+i \infty} \frac{e^{-t s}}{s^{1+p}} \Phi_{n}(s) \mathrm{d} s=\frac{1}{2 \Gamma(1+p)} \sum_{x_{k, n} \geq t^{2}} A_{k, n}\left(x_{k, n}^{1 / 2}-t\right)^{p}
$$

and

$$
\frac{1}{2 \pi i} \int_{a-i \infty}^{a+i \infty} \frac{e^{-t s}}{s^{1+p}} \Phi(s) \mathrm{d} s=\frac{1}{2 \Gamma(1+p)} \int_{t^{2}}^{\infty}\left(x^{1 / 2}-t\right)^{p} \omega(x) \mathrm{d} x .
$$

Thus, by the uniform convergence of the sequence $\left\{\Phi_{n}(s)\right\}$ in $(0, b) \times \mathbb{R}$, it follows that for any positive $t$ and $p$

$$
\lim _{n \rightarrow \infty} \sum_{x_{k, n} \geq t^{2}} A_{k, n}\left(x_{k, n}^{1 / 2}-t\right)^{p}=\int_{t^{2}}^{\infty}\left(x^{1 / 2}-t\right)^{p} \omega(x) \mathrm{d} x
$$


Next we consider, for a positive integer $n$, the function

$$
f_{n}(t, p)=\sum_{x_{k, n} \geq t^{2}} A_{k, n}\left(x_{k, n}^{1 / 2}-t\right)^{p}
$$

of the variable $p$, with $t$ a parameter. If $0 \leq p \leq 1$, we have

$$
\left|x_{k, n}^{1 / 2}-t\right|^{p} \leq\left(\left|x_{k, n}^{1 / 2}\right|+|t|\right)^{p} \leq \begin{cases}\left|x_{k, n}^{1 / 2}\right|+|t|, & \text { if }\left|x_{k, n}^{1 / 2}\right|+|t|>1 \\ 1, & \text { if }\left|x_{k, n}^{1 / 2}\right|+|t| \leq 1\end{cases}
$$

Hence, we define

$$
I_{1}=\left\{k: x_{k, n} \geq t^{2}, x_{k, n}^{1 / 2}+|t|>1\right\} \quad \text { and } \quad I_{2}=\left\{k: x_{k, n} \geq t^{2}, x_{k, n}^{1 / 2}+|t| \leq 1\right\},
$$

so that

$$
\left|f_{n}(t, p)\right| \leq \sum_{k \in I_{1}} A_{k, n}\left(x_{k, n}^{1 / 2}+|t|\right)+\sum_{k \in I_{2}} A_{k, n} \leq \sum_{k} A_{k, n} x_{k, n}^{1 / 2}+(|t|+1) \sum_{k} A_{k, n} .
$$

But

$$
\sum_{k=1}^{n} A_{k, n} x_{k, n}^{1 / 2} \leq\left(\sum_{k=1}^{n} A_{k, n} x_{k, n}\right)^{1 / 2}\left(\sum_{k=1}^{n} A_{k, n}\right)^{1 / 2} \leq\left(c_{1} c_{0}\right)^{1 / 2} .
$$

Thus for arbitrary $t>0$, but fixed, we have

$$
\left|f_{n}(t, p)\right| \leq\left(c_{0} c_{1}\right)^{1 / 2}+(1+t) c_{0}, \quad \forall n \geq 1 .
$$

By (3.7) and (3.8), we see that $f_{n}(t, p)$ converges uniformly with respect to $p$ on $[0,1]$ to

$$
f(t, p)=\int_{t^{2}}^{\infty}\left(x^{1 / 2}-t\right)^{p} \omega(x) \mathrm{d} x
$$

Therefore,

$$
\lim _{p \rightarrow 0+}\left(\lim _{n \rightarrow \infty} f_{n}(t, p)\right)=\lim _{n \rightarrow \infty}\left(\lim _{p \rightarrow 0+} f_{n}(t, p)\right)=f(t, 0),
$$

or, equivalently,

$$
\lim _{n \rightarrow \infty} \sum_{x_{k, n} \geq t^{2}} A_{k, n}=\int_{t^{2}}^{\infty} \omega(x) \mathrm{d} x .
$$

So, for any $\alpha, \beta>0$, we conclude that

$$
\lim _{n \rightarrow \infty} \sum_{\alpha \leq x_{k, n} \leq \beta} A_{k, n}=\int_{\alpha}^{\beta} \omega(x) \mathrm{d} x>0 .
$$

From (3.9), we see that for sufficiently large $n$, and for any given interval $(\alpha, \beta)$ in $\mathbb{R}_{+}$, we can always find nodes $x_{j, n}$ inside that interval.

Finally, by (3.9), we also see that the sequence of quadrature formulas $\left\{I_{n}(f)\right\}_{n=1}^{\infty}$ converges for any characteristic function $f$, which implies convergence for any function $f$ for which $f(x) \omega(x)$ is integrable on $[0, \infty)$. Therefore, the proof follows by Theorem 3.1.

It is a direct consequence of Theorems 3.1 and 3.4 that 
Corollary 3.5. With the same notation and under the same conditions as in Theorem 3.3, we have

$$
\lim _{n \rightarrow \infty} I_{n}(f)=I_{\omega}(f)
$$

for any function $f$ for which $f(x) \omega(x)$ is integrable on $[0, \infty)$.

Now we are in a position to give our main results about the class of functions $f$ for which the Gauss-type quadrature formulas converge if the moments satisfy either (3.1) or (3.2).

Theorem 3.6. Let $\omega$ be a weight function on $[0, \infty)$ whose moments satisfy (3.1), i.e.

$$
c_{n} \leq C \Gamma(\gamma(n+1+\theta)) R^{\gamma n}, \quad n \in \mathbb{N},
$$

with $\theta>-1, C, R \in \mathbb{R}_{+}$, and $0<\gamma \leq 2$. Let $\{p(n)\}$ be a sequence of integers such that $0 \leq p(n) \leq 2 n-1$, and define $q(n)=2 n-p(n)$. Assume that $\lim _{n \rightarrow \infty} q(n)=$ $\infty$. Let $\left\{I_{n}(f)\right\}_{1}^{\infty}$ be the sequence of Gauss-type formulas in $\Lambda_{-p(n), q(n)-1}, n=$ $1,2, \ldots$ Then, for any locally integrable function $f$ satisfying for sufficiently large $x$

$$
|f(x)| \leq \frac{e^{r x^{1 / \gamma}}}{x^{1+\theta+\rho}}, \quad 0<r<1 / R, \quad 0<\rho<1, \quad \theta>-1
$$

we have

$$
\lim _{n \rightarrow \infty} I_{n}(f)=I_{\omega}(f) .
$$

Proof. Let us consider the Mittag-Leffler function $E_{\gamma}(y)$, as given in (3.3). Our first aim is to prove that the integral

$$
I_{\omega}\left(\frac{E_{\gamma}\left(s^{\gamma} x\right)}{1+x^{1+\theta+\rho}}\right), \quad s \in \mathbb{C}, \quad|s|<1 / R,
$$

exists. Recall from (3.3) that

$$
E_{\gamma}\left(s^{\gamma} x\right)=\sum_{l=0}^{\infty} \frac{s^{\gamma l} x^{l}}{\Gamma(\gamma l+1)}
$$

Take $b>0$; then by the uniform convergence of the series 3.11) on $[0, b]$ we can write

$$
\begin{aligned}
\int_{0}^{b} \frac{\left|E_{\gamma}\left(s^{\gamma} x\right)\right|}{1+x^{1+\theta+\rho}} \omega(x) \mathrm{d} x & \leq \lim _{n \rightarrow \infty} \sum_{l=0}^{n} \frac{|s|^{\gamma l}}{\Gamma(\gamma l+1)} \int_{0}^{b} \frac{x^{l}}{1+x^{1+\theta+\rho}} \omega(x) \mathrm{d} x \\
& =\sum_{l=0}^{\infty} \frac{|s|^{\gamma l}}{\Gamma(\gamma l+1)} \int_{0}^{b} \frac{x^{l}}{1+x^{1+\theta+\rho}} \omega(x) \mathrm{d} x \\
& \leq \sum_{l=0}^{\infty} \frac{|s|^{\gamma l}}{\Gamma(\gamma l+1)} \int_{0}^{\infty} \frac{x^{l}}{1+x^{1+\theta+\rho}} \omega(x) \mathrm{d} x
\end{aligned}
$$


But the series (3.12) is convergent for any complex $s$ in $|s|<1 / R$. Indeed, with $\delta=1+\rho+\theta>0$ we have

$$
\begin{aligned}
\int_{0}^{\infty} \frac{x^{l}}{1+x^{1+\theta+\rho}} \omega(x) \mathrm{d} x & \leq \int_{0}^{\infty} x^{l-\delta} \omega(x) \mathrm{d} x \\
& =\int_{0}^{1} \frac{x^{l}}{x^{\delta}} \omega(x) \mathrm{d} x+\int_{1}^{\infty} \frac{x^{l}}{x^{\delta}} \omega(x) \mathrm{d} x \\
& \leq \int_{0}^{1} \frac{\omega(x)}{x^{\delta}} \mathrm{d} x+\int_{1}^{\infty} x^{l} \omega(x) \mathrm{d} x .
\end{aligned}
$$

Take $p \in \mathbb{N}$ such that $p>\delta$. Then $x^{\delta} \geq x^{p}$ for all $x \in[0,1]$, thus

$$
\int_{0}^{\infty} \frac{x^{l}}{1+x^{1+\theta+\rho}} \omega(x) \mathrm{d} x \leq \int_{0}^{1} x^{-p} \omega(x) \mathrm{d} x+\int_{1}^{\infty} x^{l} \omega(x) \mathrm{d} x \leq c_{-p}+c_{l}=A+c_{l}
$$

with $A$ a constant. Therefore, the series (3.12) is less than or equal to

$$
\begin{aligned}
\sum_{l=0}^{\infty} \frac{|s|^{\gamma l}}{\Gamma(\gamma l+1)}\left[A+c_{l}\right] & =A \sum_{l=0}^{\infty} \frac{|s|^{\gamma l}}{\Gamma(\gamma l+1)}+\sum_{l=0}^{\infty} \frac{c_{l}|s|^{\gamma l}}{\Gamma(\gamma l+1)} \\
& =E_{\gamma}\left(|s|^{\gamma}\right)+\sum_{l=0}^{\infty} \frac{c_{l}|s|^{\gamma l}}{\Gamma(\gamma l+1)} .
\end{aligned}
$$

Now by (3.1)

$$
\sum_{l=0}^{\infty} \frac{c_{l}|s|^{\gamma l}}{\Gamma(\gamma l+1)} \leq C \sum_{l=0}^{\infty} \frac{\Gamma(\gamma(l+1+\theta))}{\Gamma(\gamma l+1)} R^{\gamma l}|s|^{\gamma l},
$$

which by (3.4) is convergent if $[R|s|]^{\gamma}<1$, i.e., if $|s|<1 / R$.

Thus it follows from (3.12) that the integral

$$
I_{\omega}\left(\frac{\left|E_{\gamma}\left(s^{\gamma} x\right)\right|}{1+x^{1+\theta+\rho}}\right), \text { hence also } I_{\omega}\left(\frac{E_{\gamma}\left(s^{\gamma} x\right)}{1+x^{1+\theta+\rho}}\right)
$$

exists. Using the asymptotic behavior for $E_{\gamma}(z)$ (see [8])

$$
E_{\gamma}(z)=\frac{1}{\gamma} \exp \left(z^{1 / \gamma}\right)+O\left(\frac{1}{|z|}\right), \quad z \rightarrow \infty \text { in }|\operatorname{Arg}(z)| \leq \gamma \pi / 2,
$$

we find that

$$
\lim _{x \rightarrow \infty}\left|\frac{\gamma E_{\gamma}(x)}{\exp \left(x^{1 / \gamma}\right)}\right|=1 .
$$

Thus, from (3.10) and (3.13) we have that $I_{\omega}(f)$ exists, and by Corollary 3.5 the proof now follows.

Remark 3.2. From the proof of Theorem 3.6 and from Theorems 3.1 and 3.4, we see that

$$
\lim _{n \rightarrow \infty} I_{n}\left(\frac{E_{\gamma}\left(s^{\gamma} x\right)}{1+x^{1+\theta+\rho}}\right)=I_{\omega}\left(\frac{E_{\gamma}\left(s^{\gamma} x\right)}{1+x^{1+\theta+\rho}}\right), \quad s \in \mathbb{C}, \quad|s|<1 / R .
$$

On the other hand, proceeding as in Theorem 3.3, it can be easily checked that convergence in (3.14) holds uniformly on compact subsets of $|s|<1 / R$.

Now if instead of condition (3.1) we have that (3.2) holds, a similar result can be proved in an analogous way. We give it for completeness. 
Theorem 3.7. Let $\omega$ be a weight function whose moments satisfy (3.2), i.e.

$$
c_{-n} \leq \hat{C} \Gamma(\gamma(n-1-\hat{\theta})) \hat{R}^{\gamma n}, \quad n \in \mathbb{N},
$$

with $\hat{\theta}<-1, \hat{C}, \hat{R} \in \mathbb{R}_{+}$, and $0<\gamma \leq 2$. Let $\{p(n)\}$ be a sequence of integers such that $0 \leq p(n) \leq 2 n-1$, and define $q(n)=2 n-p(n)$. Assume that $\lim _{n \rightarrow \infty} p(n)=$ $\infty$. Let $\left\{I_{n}(f)\right\}_{1}^{\infty}$ be the sequence of Gauss-type formulas in $\Lambda_{-p(n), q(n)-1}, n=$ $1,2, \ldots$ Then, for any locally integrable function $f$ on $[a, \infty)(a>0)$ satisfying for sufficiently small $x$

$$
|f(x)| \leq \frac{e^{\hat{r} / x^{\gamma}}}{x^{1+\hat{\theta}-\rho}}, \quad \hat{r}<1 / R, \quad 0<\rho<1, \quad \hat{\theta}<-1,
$$

we have

$$
\lim _{n \rightarrow \infty} I_{n}(f)=I_{\omega}(f) .
$$

Remark 3.3. Recall that by setting $p(n)=0$ for all $n=1,2, \ldots$, we recover the classical Gaussian quadrature formulas.

If we set $\gamma=2$ and $\theta=0$, we obtain the convergence result of Uspensky [24].

Other results for the convergence of Gauss-type formulas in $\Lambda_{-p, 2 n-p-1}$ were given in [10] for the "balanced" situation, i.e. for $p=n$ and for $\gamma=2$ and $\theta=0$.

We also note that the weight functions studied by López-Lagomasino and Martínez-Finkelshtein in [15] are of the form

$$
\omega(x)=x^{\alpha} e^{-(\tau(x))}
$$

such that

$$
\lim _{x \rightarrow 0+}(s x)^{\gamma_{1}} \tau(x)=\lim _{x \rightarrow+\infty}(s x)^{-\gamma_{2}} \tau(x)=A>0,
$$

where $\alpha \in \mathbb{R}, s>0$, with $\gamma_{1}>1 / 2$ and $\gamma_{2}>1 / 2$. These weights satisfy both (3.1) and (3.2) for appropriate choices of $\theta$ and $\hat{\theta}$ (see Section 4).

\section{NumericAl Results}

Let us now illustrate this with some numerical examples. We restrict ourselves to the weight function

$$
\omega(x)=\frac{e^{-(x+1 / x)}}{\sqrt{x}}, \quad x \in(0, \infty) .
$$

It is a special case of (3.15)-(3.16) and therefore is included in the class mentioned in Remark [3.3. We first prove that the weight functions considered by LópezLagomasino satisfy (3.1) and (3.2). Take

$$
h(x)=x^{\gamma_{2}}+\frac{1}{x^{\gamma_{1}}}, \quad \gamma_{1}, \gamma_{2} \geq \frac{1}{2} ;
$$

then by (3.15)-(3.16), there exist positive real numbers $r, R$ and $D$ such that

$$
\exp (-\tau(x)) \leq \exp (-D h(x)), \quad \forall x \in(0, r) \cup(R, \infty) .
$$

For each integer $k$, consider the moments

$$
d_{k}=\int_{0}^{\infty} x^{k} x^{\alpha} \exp (-\tau(x)) \mathrm{d} x
$$


These $d_{k}$ satisfy (3.1) and (3.2), since indeed for $0<r<1<R$,

$d_{k}=\int_{0}^{r} x^{k} x^{\alpha} \exp (-\tau(x)) \mathrm{d} x+\int_{r}^{R} x^{k} x^{\alpha} \exp (-\tau(x)) \mathrm{d} x+\int_{R}^{\infty} x^{k} x^{\alpha} \exp (-\tau(x)) \mathrm{d} x$, which yields

$$
d_{k} \leq 2 \int_{0}^{\infty} x^{k} x^{\alpha} \exp (-D h(x)) \mathrm{d} x+F_{k}(R, r)
$$

where

$$
F_{k}(R, r)= \begin{cases}d_{0} R^{\alpha+k}, & \alpha+k \geq 0, \\ d_{0} r^{\alpha+k}, & \alpha+k<0 .\end{cases}
$$

Setting

$$
c_{k}=\int_{0}^{\infty} x^{k} x^{\alpha} \exp \left(-D\left(x^{\gamma_{2}}+\frac{1}{x^{\gamma_{1}}}\right)\right) \mathrm{d} x,
$$

we see by (4.4) that it suffices to check that the moments $\left\{c_{k}\right\}$ satisfy (3.1) and (3.2). For the sake of simplicity, we assume that $D=1$ and $\gamma_{1}=\gamma_{2}=\gamma$, i.e.

$$
c_{k}=\int_{0}^{\infty} x^{k} x^{\alpha} \exp \left(-\left(x^{\gamma}+\frac{1}{x^{\gamma}}\right)\right) \mathrm{d} x, \quad \alpha \in \mathbb{R}, \quad \gamma \geq 1 / 2 .
$$

First, we consider the moments $c_{k}$ with $k \in \mathbb{N}$. We distinguish between two cases.

1. $\alpha>-1$. Then

$$
c_{k}=\int_{0}^{\infty} x^{k} x^{\alpha} e^{-x^{\gamma}} e^{-1 / x^{\gamma}} \mathrm{d} x \leq \int_{0}^{\infty} x^{k} x^{\alpha} e^{-x^{\gamma}} \mathrm{d} x .
$$

Setting $x^{\gamma}=t, x=t^{1 / \gamma}, \mathrm{d} x=\gamma^{-1} t^{1 / \gamma-1} \mathrm{~d} t$, we get

$$
c_{k} \leq \frac{1}{\gamma} \int_{0}^{\infty} t^{\gamma^{-1}(k+\alpha+1)-1} e^{-t} \mathrm{~d} t=\frac{1}{\gamma} \Gamma\left(\gamma^{-1}(k+\alpha+1)\right) .
$$

2. $\alpha \leq-1$. For an arbitrary given $\epsilon>0$, we can find $\rho \geq 0$ such that $\alpha+\rho=$ $-1+\epsilon$, so that we have

$$
c_{k}=\int_{0}^{\infty} x^{k} x^{\alpha+\rho} e^{-x^{\gamma}} \frac{e^{-1 / x^{\gamma}}}{x^{\rho}} \mathrm{d} x .
$$

Now $x^{-\rho} e^{-1 / x^{\gamma}} \leq K$ for all $x \in[0, \infty)$, and therefore

$$
c_{k} \leq \frac{K}{\gamma} \Gamma\left(\gamma^{-1}(k+\epsilon)\right)=\frac{K}{\gamma} \Gamma\left(\gamma^{-1}(k+\theta+1)\right)
$$

with $\theta \in \mathbb{R}$ such that $\epsilon=1+\theta$, hence $\theta>-1$.

Next, let us consider the moments $c_{-k}$ with $k \in \mathbb{N}$, i.e., with the sucstitution $x=t^{-1}$,

$$
c_{-k}=\int_{0}^{\infty} x^{-k} x^{\alpha} e^{-\left(x^{\gamma}+1 / x^{\gamma}\right)} \mathrm{d} x=\int_{0}^{\infty} t^{k-2-\alpha} e^{-\left(t^{\gamma}+1 / t^{\gamma}\right)} \mathrm{d} t .
$$

Again we distinguish between two cases.

1. $\alpha<-1$. Then

$$
c_{-k} \leq \int_{0}^{\infty} t^{k-2-\alpha} e^{-t^{\gamma}} \mathrm{d} t=\gamma^{-1} \Gamma\left(\gamma^{-1}(k-1-\alpha)\right) .
$$

Thus, it is sufficient to take $\theta=\alpha$ and thus $\theta<-1$. 
2. $\alpha \geq-1$. Set $\delta=-(\alpha+2) \leq-1$; then

$$
c_{-k}=\int_{0}^{\infty} t^{k+\delta} e^{-\left(t^{\gamma}+1 / t^{\gamma}\right)} \mathrm{d} t .
$$

Now, given $\epsilon>0$, there exists a $\rho \geq 0$ such that $\delta+\rho=-1+\epsilon$. Therefore

$$
c_{-k} \leq K \int_{0}^{\infty} t^{k} t^{-1+\epsilon} e^{-t^{\gamma}} \mathrm{d} t=\frac{K}{\gamma} \Gamma\left(\gamma^{-1}(k+\epsilon)\right),
$$

where $K$ is a positive constant. Since $\epsilon$ is arbitrary, we can take $\epsilon=-1-\hat{\theta}$ with $\hat{\theta}<-1$, so that it finally results that

$$
c_{-k} \leq \frac{K}{\gamma} \Gamma\left(\gamma^{-1}(k+\hat{\theta}-1)\right), \quad \hat{\theta}<-1 .
$$

Thus all the convergence results of Section 3 can be applied to the weight function (3.15)-3.16), and in particular to the one given in (4.1).

In order to compute the moments $c_{k}$, let us introduce the auxiliary weight function studied by Ranga [20]:

$$
\tilde{\omega}(x)=\frac{e^{-1 / 2(x+a / x)}}{\sqrt{x}}, \quad a \in \mathbb{R}, a>0, x \in(0, \infty),
$$

and set

$$
\tilde{c}_{n, a}=\int_{0}^{\infty} x^{n} \tilde{\omega}(x) \mathrm{d} x, \quad n \in \mathbb{Z}
$$

It can be checked that

$$
c_{n}=\frac{1}{2^{n+1 / 2}} \tilde{c}_{n, 4}, \quad n \in \mathbb{Z} .
$$

On the other hand, it is known that the sequence $\left\{\tilde{c}_{n, 4}\right\}$ satisfies [20]

$$
\tilde{c}_{0,4}=\frac{\sqrt{2 \pi}}{e^{2}}, \quad \tilde{c}_{-(n+1), 4}=\frac{\tilde{c}_{n, 4}}{2^{2 n+1}}, \quad \tilde{c}_{n+1,4}-(2 n+1) \tilde{c}_{n, 4}-4 \tilde{c}_{n-1,4}=0, \quad n \geq 1,
$$

or explicitly

$$
\tilde{c}_{n, 4}=\frac{\sqrt{2 \pi}}{2^{3 n} e^{2}}\left[\sum_{r=0}^{n}\left(\begin{array}{l}
2 n+1 \\
2 r+1
\end{array}\right) \sum_{s=0}^{r}\left(\begin{array}{l}
r \\
s
\end{array}\right) 2^{6 s} \frac{(2 n-2 s) !}{(n-s) !}\right], \quad n \geq 0 .
$$

In short, from (4.6) and (4.7) or 4.8), we see that the moments $\left\{c_{k}\right\}$ can be readily computed.

Now we want to estimate the integral

$$
I_{\omega}(f)=\int_{0}^{\infty} f(x) \frac{e^{-(x+1 / x)}}{\sqrt{x}} \mathrm{~d} x
$$

for different choices of the integrands $f(x)$. We shall use Gauss-type quadrature formulas $I_{n}(f)$ for $\Lambda_{-p(n), q(n)-1}$ with $p(n)$ and $q(n)$ nonnegative integers such that $p(n)+q(n)=2 n$. In the sequel, we restrict our attention to the "balanced" situation where $p(n)=n$ for all $n \in \mathbb{N}$, so that the resulting quadrature formula is exact in $\Lambda_{-n, n-1}$ for $n \in \mathbb{N}$. 
The nodes $\left\{x_{j, n}\right\}$ are the zeros of $Q_{n}(x)$, the (monic) orthogonal polynomial of degree $n$ with respect to the varying distribution $x^{-n} \omega(x) \mathrm{d} x$. From [20], we get the following three-term recurrence relation for the sequence $\left\{Q_{n}\right\}$ :

$$
Q_{n+1}(x)=(x-1) Q_{n}(x)-\frac{n}{2} x Q_{n-1}(x), \quad n \geq 1,
$$

with initial conditions $Q_{0}(x) \equiv 1$ and $Q_{1}(x)=x-1$. Let us consider the $[n / n]$ 2PA for $F_{\omega}(z)=\int_{0}^{\infty} \frac{\omega(x)}{z-x} \mathrm{~d} x$; then it is known that $[n / n]_{F_{\omega}}(z)=P_{n-1}(z) / Q_{n}(z)$, with $P_{n-1} \in \Pi_{n-1}$, and in this case it can be proved that $P_{n}$ satisfies the same recurrence as $Q_{n+1}$ (see [20]), i.e.,

$$
P_{n}(x)=(x-1) P_{n-1}(x)-\frac{n}{2} x P_{n-2}(x), \quad n \geq 1,
$$

with $P_{-1} \equiv 0$ and $P_{0}=c_{0}$.

Now take into account that

$$
[n / n]_{F_{\omega}}(z)=\frac{P_{n-1}(z)}{Q_{n}(z)}=\sum_{j=1}^{n} \frac{A_{j, n}}{z-x_{j, n}} .
$$

Then one has

$$
A_{j, n}=\frac{P_{n-1}\left(x_{j, n}\right)}{Q_{n}^{\prime}\left(x_{j, n}\right)}, \quad j=1, \ldots, n .
$$

Thus, we see that our Gauss-type formulas can be easily computed.

We shall compare these Gauss-type formulas with the classical Gauss formulas. Therefore, we write

$I_{\omega}(f)=\int_{0}^{\infty} f(x) \frac{e^{-(x+1 / x)}}{\sqrt{x}} \mathrm{~d} x=\int_{0}^{\infty} f(x) e^{-1 / x} \frac{e^{-x}}{\sqrt{x}} \mathrm{~d} x=\int_{0}^{\infty} g(x) \beta(x) \mathrm{d} x=I_{\beta}(g)$, with $g(x)=f(x) \exp (-1 / x)$ and $\beta(x)=\exp (-x) / \sqrt{x}$. Thus, the integral $I_{\beta}(g)$ will be estimated by means of the Gauss-Laguerre quadrature formula of order $-1 / 2$. We shall denote this formula as

$$
\tilde{I}_{n}(g)=\sum_{j=1}^{n} \lambda_{j, n} g\left(t_{j, n}\right) .
$$

It is well known that this formula is exact for all integrands $g \in \Pi_{2 n-1}$ while our Gauss-type formula is exact for all $f \in \Lambda_{-n, n-1}$. The construction of the Gauss-Laguerre formulas $\tilde{I}_{n}(g)$ require the moments $\delta_{0}, \ldots, \delta_{2 n-1}$ with $\delta_{j}=$ $\int_{0}^{\infty}\left(x^{j} \exp (-x) / \sqrt{x}\right) \mathrm{d} x$, while the Gauss-type formulas $I_{n}(f)$ require the moments $c_{-n}, \ldots, c_{n-1}$. We will see that the inclusion of the moments $c_{k}$ both with positive and with negative index yields excellent numerical results.

We shall take for $f(x)$ the following functions:

\begin{tabular}{c|c}
$i$ & $f_{i}(x)$ \\
\hline 1 & $x^{-1}$ \\
2 & $\left(x^{2}-1\right) x^{-1} \log (x)$ \\
3 & $x^{-1 / 2} \log (x)$ \\
4 & $\exp (-\log (x) / \sqrt{x})$ \\
5 & $\sin x$ \\
6 & $x^{-3 / 2} e^{x} / \log (1+x)$ \\
7 & $\log (1+x) e^{1 / x}$
\end{tabular}



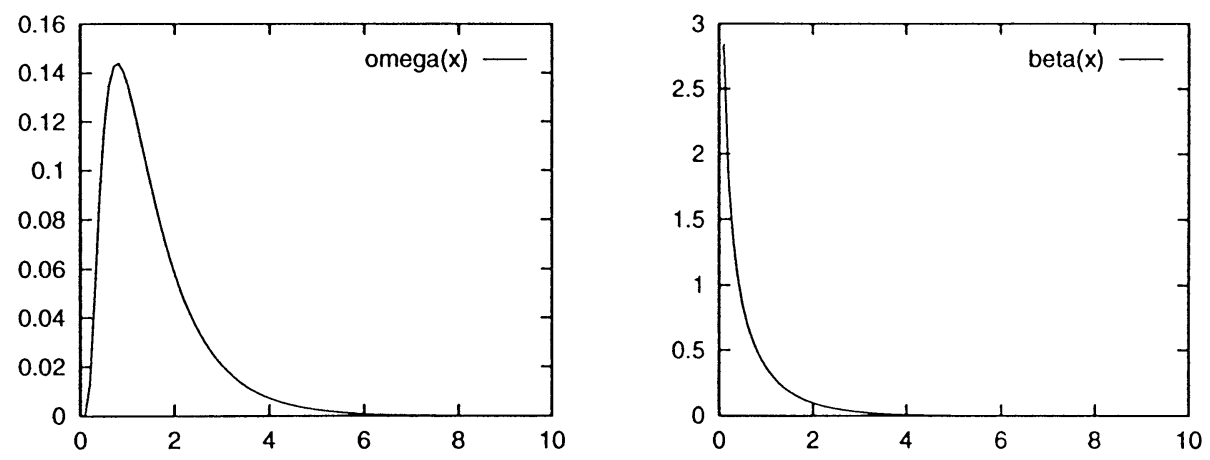

Figure 2. The weights $\omega(x)=\exp (-(x+1 / x))$ and $\beta(x)=\exp (-x) / \sqrt{x}$

In Figure 2 we have plotted the weight functions $\omega(x)=x^{-1 / 2} \exp (-(x+1 / x))$ and $\beta(x)=x^{-1 / 2} \exp (-x)$. In Figures 3 .6 (on the following pages) one can find the plots of the integrands $f_{i}(x)$ of the table above, the functions $g_{i}(x)=f_{i}(x) \exp (-1 / x)$ and the functions $h_{i}(x)=\omega(x) f_{i}(x)=g_{i}(x) \beta(x)$.

In Figure 7 we plot, as a function of $n=1, \ldots, 10$, the absolute values of the absolute errors of the Gauss-Laguerre and the Gauss-type formulas of this paper. The solid line corresponds to the classical Gauss formulas, while the dotted line corresponds to the Gauss-type formulas of this paper.

In the example $f_{6}$ we note that $\lim _{x \rightarrow 0^{+}} f(x)=\infty$ and $\lim _{x \rightarrow \infty} f(x)=\infty$. It is at the "boundary" of what can be integrated, because for the function

$$
f_{\epsilon}(x)=\frac{e^{x}}{\log (1+x) x^{3 / 2+\epsilon}}, \quad \epsilon>0,
$$

the integral $I_{\omega}\left(f_{\epsilon}\right)$ is divergent. By our quadrature rules we do find convergence, but it is extremely slow.

For the example $f_{7}$, it should be noted that the factor $e^{1 / x}$ is "absorbed" by the weight. In fact, the integral reduces to

$$
I_{\omega}\left(f_{7}\right)=\int_{0}^{\infty} \log (1+x) \frac{e^{-x}}{\sqrt{x}} \mathrm{~d} x,
$$

and of course, this integral can be treated perfectly well by a classical GaussLaguerre quadrature formula. The Gauss-type formulas of this paper are obviously less appropriate. They still converge, but they are outperformed by the GaussLaguerre formulas.

For $f_{1}$ and $f_{3}$, the errors for the Gauss-type formulas of this paper are zero.

There is an explanation for the surprising exactness. Observe that $x^{-1} \in$ $\Lambda_{-n, n-1}$ for any $n \geq 1$. This is the explanation for $f_{1}$. The explanation for $f_{3}$ is as follows. We have

$$
\begin{aligned}
I & =I_{\omega}(f)=\int_{0}^{\infty} f(x) \omega(x) \mathrm{d} x=\int_{0}^{\infty} \frac{\log x}{x} \exp (-(x+1 / x)) \mathrm{d} x \\
& =\int_{0}^{1} \frac{\log x}{x} \exp (-(x+1 / x)) \mathrm{d} x+\int_{1}^{\infty} \frac{\log x}{x} \exp (-(x+1 / x)) \mathrm{d} x=I_{1}+I_{2} .
\end{aligned}
$$





Figure 3. The integrands $f_{i}, g_{i}(x)=\exp (-1 / x) f_{i}(x), h_{i}(x)=$ $f_{i}(x) \omega(x)=g_{i}(x) \beta(x), i=1,2$

Making the change of variable $x=1 / t$ in $I_{2}$, we see that $I_{2}=-I_{1}$, so that $I=0$. Let us now explain why $I_{n}(f)=0$ for all $n \in \mathbb{N}$. This is partially due to a certain symmetry in the weight function $\omega(x)=x^{-1 / 2} \exp (-(x+1 / x))$. In general, for $c>0$, it is said that $\omega(x)$ is $c$-inversive on $(0, \infty)[19]$ if

$$
\omega(c / x)=\frac{x}{\sqrt{c}} \omega(x), \quad \forall x \in(0, \infty) .
$$

In [19] we can find the following result. 

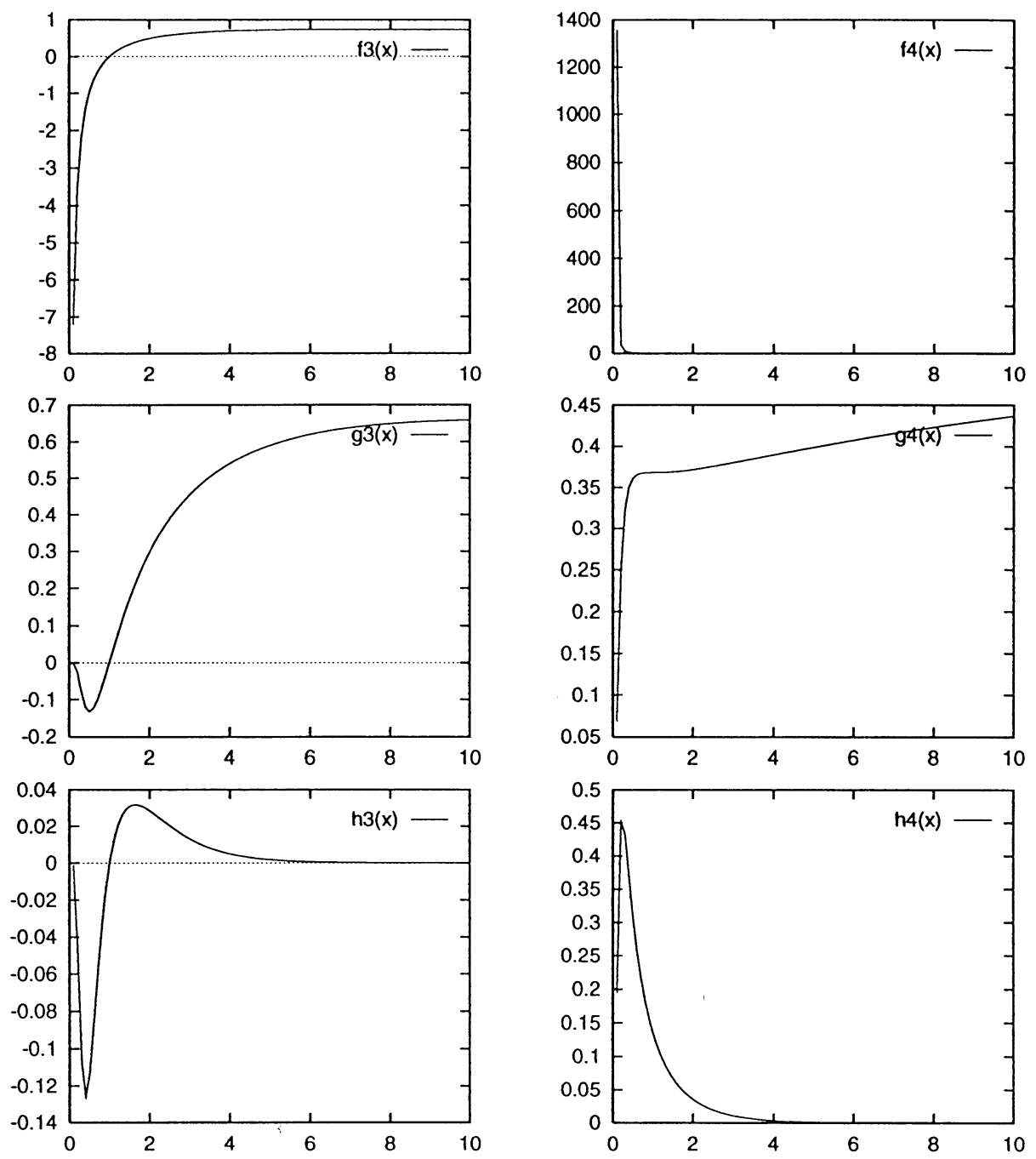

Figure 4. The integrands $f_{i}, g_{i}(x)=\exp (-1 / x) f_{i}(x), h_{i}(x)=$ $f_{i}(x) \omega(x), i=3,4$

Theorem 4.1. Let $I_{n}(f)=\sum_{j=1}^{n} A_{j, n} f\left(x_{j, n}\right)$ be the n-point Gauss-type formula in $\Lambda_{-n, n-1}$ with respect to a c-inversive weight $\omega(x)$. Then

$$
\begin{gathered}
x_{j, n}=\frac{c}{x_{n+1-j}}, \quad j=1, \ldots,\left\lfloor\frac{n+1}{2}\right\rfloor, \quad n \geq 1, \\
\frac{A_{j, n}}{\sqrt{x_{j, n}}}=\frac{A_{n+1-j, n}}{\sqrt{x_{n+1-j, n}}}, \quad j=1, \ldots,\left\lfloor\frac{n+1}{2}\right\rfloor, \quad n \geq 1,
\end{gathered}
$$

where $\lfloor x\rfloor$ is the integer part of $x$. 



FiguRE 5. The integrands $f_{i}, g_{i}(x)=\exp (-1 / x) f_{i}(x), h_{i}(x)=$ $f_{i}(x) \omega(x)=g_{i}(x) \beta(x), i=5,6$

Clearly our weight function (4.1) is $c$-inversive with $c=1$. Hence, the nodes of the corresponding quadrature formula verify

$$
x_{n+1-j, n}=1 / x_{j, n}, \quad j=1, \ldots,\left\lfloor\frac{n+1}{2}\right\rfloor .
$$

Furthermore, when $n$ is odd, say $n=2 k+1$, then $x_{k+1, n}=1$. On the other hand,

$$
A_{n+1-j, n}=\frac{A_{j, n}}{x_{j, n}}, \quad j=1, \ldots,\left\lfloor\frac{n+1}{2}\right\rfloor,
$$

and since $f(x)=x^{-1 / 2} \log x$, we have $f(1 / x)=-x f(x)$. Therefore, we get for $I_{n}(f)$ the following results: 

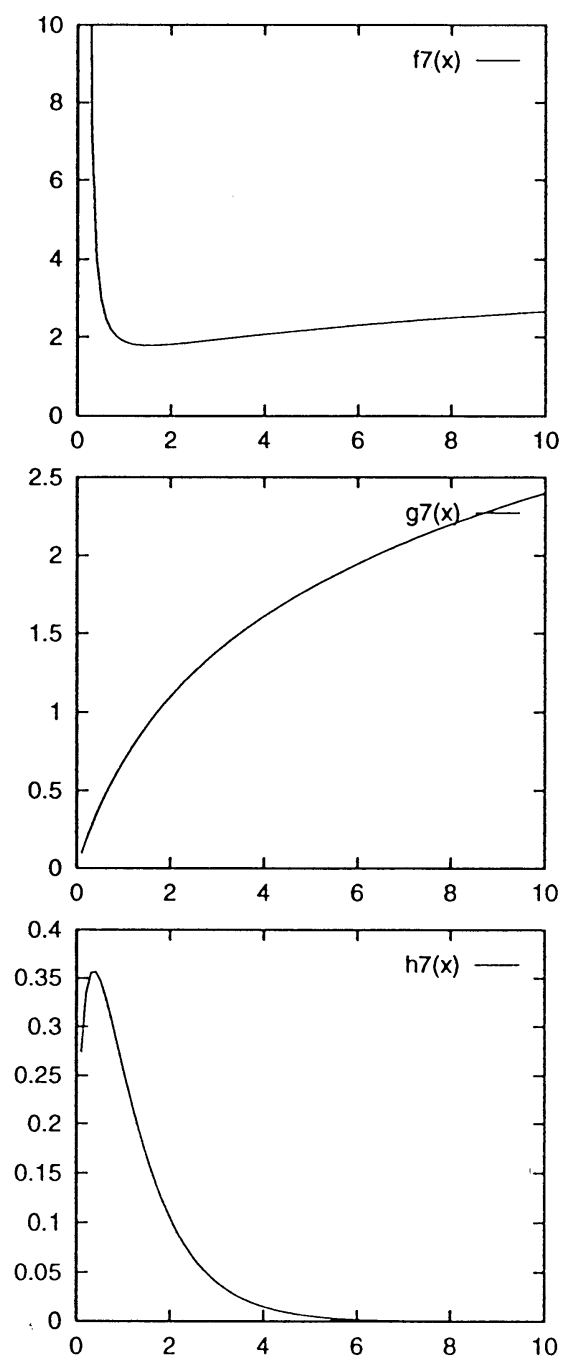

Figure 6 . The integrands $f_{i}, g_{i}(x)=\exp (-1 / x) f_{i}(x), h_{i}(x)=$ $f_{i}(x) \omega(x)=g_{i}(x) \beta(x), i=7$ 

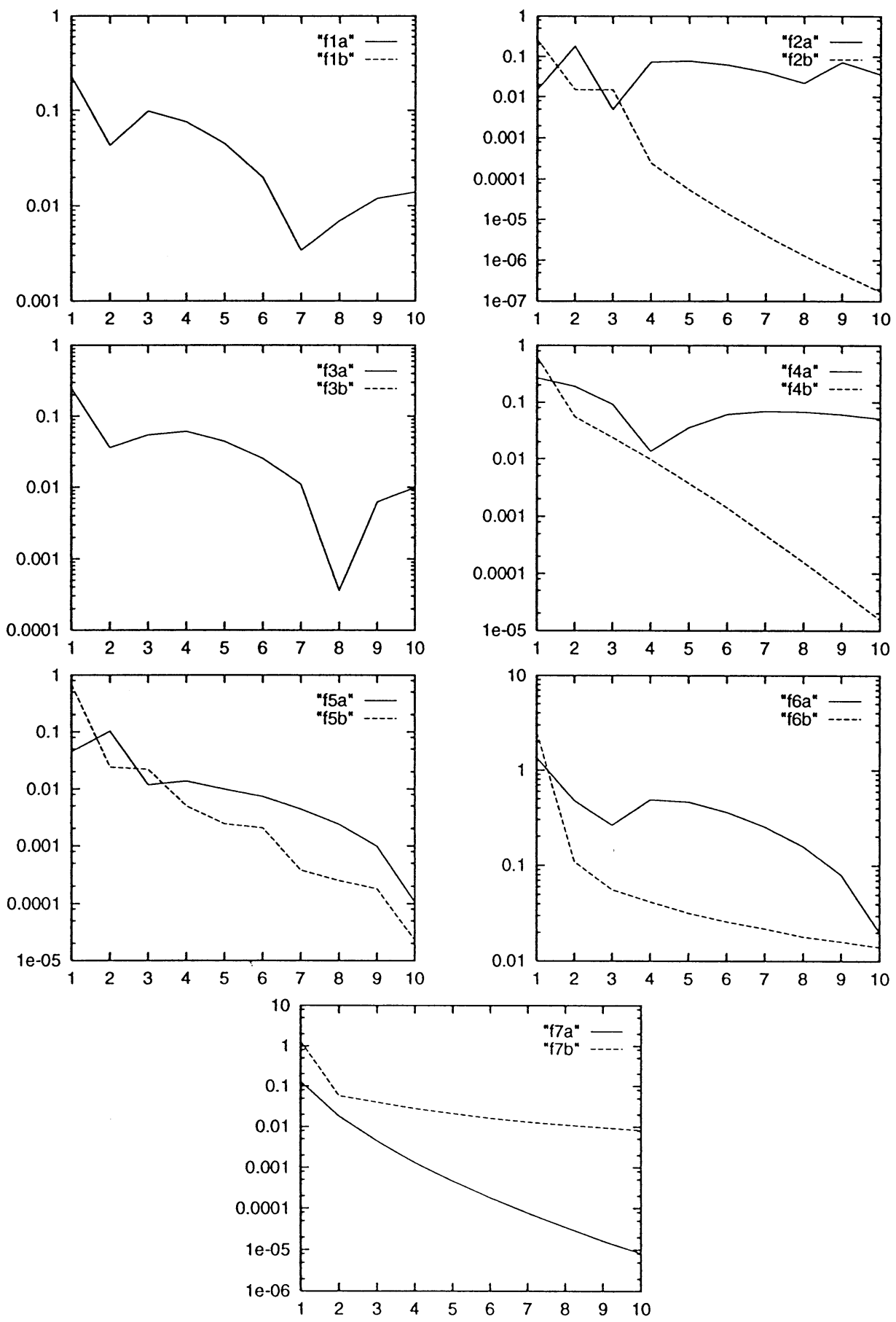

FiguRE 7. Errors as a function of $n:\left|I_{\omega}\left(f_{i}\right)-I_{n}\left(f_{i}\right)\right|$ (dotted line) and $\left|I_{\beta}\left(g_{i}\right)-\tilde{I}_{n}\left(g_{i}\right)\right|$ (solid line) 

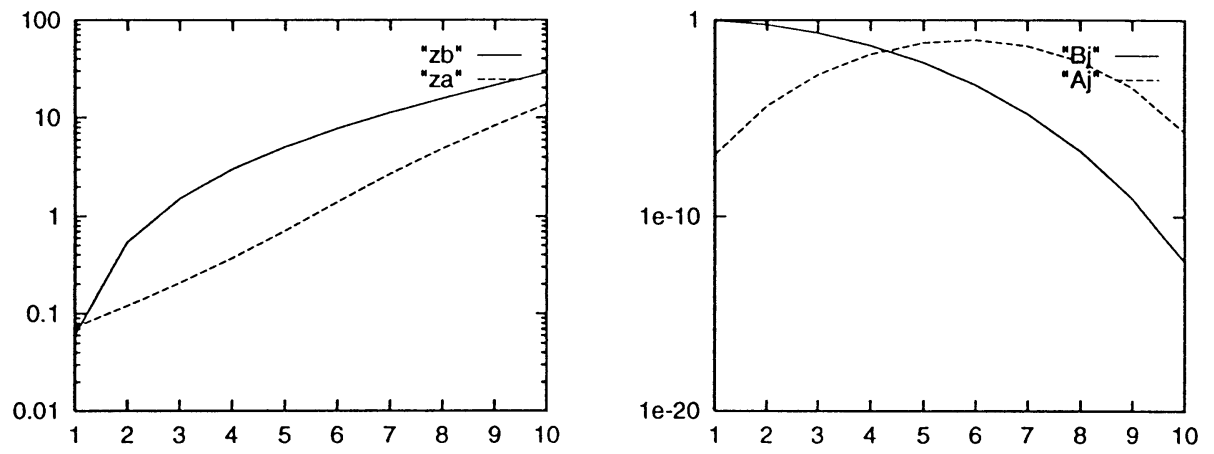

Figure 8. Zeros and weights for 10 point formulas of GaussLaguerre type (solid line) and Gauss-type formula of this paper (dotted line)

1. $n=2 k$ :

$$
\begin{aligned}
I_{2 k}(f) & =\sum_{j=1}^{k} A_{j, n} f\left(x_{j, n}\right)+\sum_{j=k+1}^{n} A_{j, n} f\left(x_{j, n}\right) \\
& =\sum_{j=1}^{k}\left[A_{j, n} f\left(x_{j, n}\right)+A_{n+1-j, n} f\left(x_{n+1-j, n}\right)\right] \\
& =\sum_{j=1}^{k}\left[A_{j, n} f\left(x_{j, n}\right)+\frac{A_{j, n}}{x_{j, n}} f\left(1 / x_{j, n}\right)\right] \\
& =\sum_{j=1}^{k}\left[A_{j, n} f\left(x_{j, n}\right)-A_{j, n} f\left(x_{j, n}\right)\right]=0 .
\end{aligned}
$$

2. $n=2 k+1$ : As is the case of an even $n$, we find that

$$
I_{2 k+1}(f)=A_{k+1, n} f\left(x_{k+1, n}\right)=A_{k+1, n} \log (1)=0 .
$$

As a general conclusion from the examples above, we may say that the Gausstype formulas $I_{n}(f)$ compete very favourable with the Gauss-Laguerre formulas, taking into account that both require the same computational effort. In our opinion, the reason for this good numerical behavior is to be found in the distribution of the nodes. In Figure 8 we have plotted in the left figure the nodes $x_{k, 10}$ as a function of $k$ for the 10-point Gauss-Laguerre quadrature (full line) and of the 10-point Gauss-type formula of this paper (dotted line). In the right figure, one can see the corresponding weights. $\mathrm{Aj}$ are the weights $A_{j, 10}$ and $\mathrm{Bj}$ are the weights of the 10-point Gauss-Laguerre formula.

As a final remark concerning the numerics, we should give a note of warning. The limitations of classical Gaussian quadrature formulas are well known. Convergence is especially slow when the integrand exhibits a non-smooth behaviour near the interval of integration. Our examples confirm this: the integrand $g(x)$ contains the factor $\exp (-1 / x)$, and when this is not compensated by a corresponding factor in $f(x)$, the convergence is slow indeed. Similar observations can be made for the Gauss-type formulas studied in this paper. In general, however, convergence is 
faster, although it is possible to design examples where this is not true (see e.g. the last one), where the above argument is in favour of the classical Gauss formula.

On the other hand, for many practical integration routines, one prefers to use so-called automatic integration rules, which may be adaptive or not and iterative or not, and which include many skillful techniques which go far beyond the kind of integration rules that we have studied in this paper and in the papers [4, 2, 3]. For further details see [7, Chap. 6] and the references therein. The Gauss-type formulas of our papers are not intended to compete with this kind of automatic integrators. Our interest in the formulas and the numerical examples is therefore mainly theoretical and because of the nice relation that exists with two-point Padé approximation. On the other hand, we do not know about experiments testing these rules in a numerical context.

\section{REFERENCES}

[1] M. Abramowitz and A. Stegun, Handbook of mathematical functions, Dover Publications, New York, 1966. MR 34:8606

[2] A. Bultheel, C. Díaz-Mendoza, P. González-Vera, and R. Orive, Quadrature on the half line and two-point Padé approximants to Stieltjes functions. Part II: Convergence, J. Comput. Appl. Math. 77 (1997), 53-76. MR 98a:41023

[3] _ Quadrature on the half line and two-point Padé approximants to Stieltjes functions. Part III: The unbounded case, J. Comput. Appl. Math. 87 (1997), 95-117. MR 98j:41033

[4] A. Bultheel, P. González-Vera, and R. Orive, Quadrature on the half line and two-point Padé approximants to Stieltjes functions. Part I: Algebraic aspects, J. Comput. Appl. Math. 65 (1995), 57-72. MR 96m:41018

[5] T. Chihara, An introduction to orthogonal polynomials, Gordon and Breach Science Publishers, New York, 1978. MR 58:1979

[6] L. Cochran and S.C. Cooper, Orthogonal Laurent polynomials on the real line, Continued fractions and orthogonal functions (New York) (S.C. Cooper and W.J. Thron, eds.), Marcel Dekker, 1994, pp. 47-100. MR 95b:42024

[7] P.J. Davis and P. Rabinowitz, Methods of numerical integration, 2nd ed., Academic Press, 1984. MR 86d:65004

[8] A. Erdelyi (ed.), Higher transcendental functions, vol. 3, Mc Graw-Hill, New York, 1955. MR 16:586c

[9] W. Gautschi, A survey of Gauss-Christoffel quadrature formulae, E.B. Christoffel. The influence of his work on mathematical and physical sciences (Basel) (P.L. Butzer and F. Feher, eds.), Birkhäuser Verlag, 1981, pp. 72-147. MR 83g:41031

[10] C. González-Concepción, P. González-Vera, and L. Casasús, On the convergence of certain quadrature formulas defined on unbounded intervals, Orthogonal Polynomials and their Applications (J. Vinuesa, ed.), Lecture Notes in Pure and Appl. Math., vol. 117, Marcel Dekker Inc., 1989, pp. 147-151. MR 91f:41037

[11] W.B. Jones, O. Njåstad, and W.J. Thron, Two-point Padé expansions for a family of analytic functions, J. Comput. Appl. Math. 9 (1983), 105-124. MR 84j:30057

[12] W.B. Jones and W.J. Thron, Orthogonal Laurent polynomials and Gaussian quadrature, Quantum mechanics in mathematics, chemistry and physics (New York) (K. Gustafson and W.P. Reinhardt, eds.), Plenum, 1984, pp. 449-455.

[13] W.B. Jones, W.J. Thron, and H. Waadeland, A strong Stieltjes moment problem, Trans. Amer. Math. Soc. 206 (1980), 503-528. MR 81j:30055

[14] V.J. Krylov, Approximate calculation of integrals, MacMillan, 1962. MR 26:2008

[15] G. López-Lagomasino and A. Martínez-Finkelshtein, Rate of convergence of two-point Padé approximants and logarithmic asymptotics of Laurent-type orthogonal polynomials, Constr. Approx. 11 (1995), 255-286. MR 96i:41016

[16] G.L. Lopes [López-Lagomasino], On the convergence of Padé approximants for Stieltjes type functions, Math. USSR-Sb. 39 (1981), 281-288. MR 81m:30034

[17] On the asymptotics of the ratio of orthogonal polynomials and convergence of multipoint Padé approximants, Math. USSR-Sb. 56 (1985), 207-219. MR 87e:30050 
[18] — Convergence of Padé approximants of Stieltjes type meromorphic functions and comparative asymptotice for orthogonal polynomials, Math. USSR-Sb. 64 (1989), 207-227. MR 90g:30003

[19] A. Sri Ranga, Another quadrature rule of highest algebraic degree, Numer. Math. 28 (1994), 283-294. MR 95c:65047.

[20] A. Sri Ranga and J.H. McCabe, On the extensions of some classical distributions, Proc. Edinburgh Math. Soc. 34 (1991), 12-29. MR 92b:30003

[21] T.J. Stieltjes, Quelques recherches sur la théorie des quadratures dites mécaniques, Ann. Acad. École Norm. Paris, Sér. 31 (1884), 409-426, Oeuvres vol. 1, pp. 377-396. MR 95g:01033

[22] , Recherches sur les fractions continues, Ann. Fac. Sci. Toulouse 8 (1894), J.1-122, 9:1-47, Enlish transl.: Oeuvres Complèts, Collected Papers, Springer Verlag, 1993, Vol. 2, 609-745. MR 95g:01033

[23] J.V. Uspensky, On the convergence of quadrature formulas between infinite limits, Bulletin of the Russian Academy of Sciences (1916).

[24] - On the convergence of quadrature formulas related to an infinite interval, Trans. Amer. Math. Soc. 30 (1928), 542-554.

Department of Computer Science, K.U. Leuven, Celestijnenlaan 200A, B-3001 HevERLEE, BELGIUM

E-mail address: Adhemar.Bultheel@cs.kuleuven.ac.be

$U R L:$ http://www.cs.kuleuven.ac.be/ ${ }^{\sim a d e /}$

Department Mathematical Analysis, Univ. La Laguna, Tenerife, Canary Islands, SPAIN

E-mail address: cjdiaz@ull.es

E-mail address: pglez@ull.es

E-mail address: rorive@ull.es 